The effect of a concentration-dependent viscosity on particle transport in a channel flow with porous walls

by

J. G. Herterich

I. M. Griffiths

R. W. Field

D. Vella 



\title{
The effect of a concentration-dependent viscosity on particle transport in a channel flow with porous walls
}

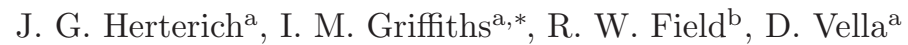 \\ ${ }^{a}$ OCCAM, Mathematical Institute, University of Oxford, 24-29 St Giles', Oxford OX1 3LB, UK \\ ${ }^{b}$ Department of Engineering Science, University of Oxford, Parks Road, Oxford OX1 3PJ, UK
}

\begin{abstract}
We analyse the transport of a dilute suspension of particles through a channel with porous walls accounting for the concentration dependence of the viscosity. Two cases of leakage flow of fluid through the porous channel walls are studied: (i) constant flux, and (ii) dependent on the pressure drop across the wall. The effect of mixing the suspension first compared with point injection is examined by considering inlet concentration distributions of different widths. We find that a pessimal distribution width exists that maximizes the required hydrodynamic pressure for a constant fluid influx. We also show that the presence of an osmotic pressure may lead to fluid being sucked into the channel. We consider how the application of an external hydrodynamic pressure affects this observation and discuss the significance of our results for water filtration.
\end{abstract}

Keywords: porous walls, concentration-dependent viscosity, water filtration, concentration polarization

\section{Introduction}

Water filtration is becoming increasingly important as a method of water treatment in our everyday lives. Many of the processes are not fully understood, including the crossflow filtration system we consider. A crossflow filtration system consists of a fluid with contaminants (often particulates) flowing tangentially to a porous membrane. This membrane allows the fluid to pass through but rejects the particulates. The filtration is driven by two pressure differences: (i) the hydrodynamic pressure difference across the membrane and (ii) the difference in osmotic pressure across the membrane. The osmotic pressure on the filtrate side is generally close to zero while that on the feed side is greater than that in the bulk flow due to the build-up of particles near the membrane surface. This phenomenon is referred to as concentration polarization and is one of the main limitations of the efficacy of water filtration [1] since the high osmotic pressure on the feed side of the membrane reduces the effective pressure that drives filtration.

The fluid velocity in a channel with porous walls has been analysed for a constant viscosity and constant leakage velocity [2]. The solution is a Poiseuille-like parabolic profile decreasing in magnitude along the channel for the axial component and a transverse component with a cubic dependence on cross-channel position that is proportional to the leakage velocity. More generally with any

\footnotetext{
* Corresponding author

Email address: ian.griffiths@maths.ox.ac.uk (I. M. Griffiths)
}

flow through the porous walls, the axial flow rate in the channel is reduced and, combined with advection by the transverse flow, results in concentration polarization [3].

A complicating factor in water filtration using membranes is the dependence of the liquid viscosity on particle concentration. The liquid viscosity is often taken as a constant in models of filtration, but in practice depends on many local properties of the fluid such as its temperature, density and shear rate. Of particular concern in this paper is the effect of concentration-dependent viscosities on crossflow filtration. Davis \& Sherwood [4] consider the convection-diffusion equation for particles in a steady-state boundary layer with a concentration-dependent viscosity and diffusivity given by Davis \& Leighton [5]. This model assumes that, outside the boundary layer, the bulk concentration of particles is constant. Physically, this may be achieved in a system in which the Péclet number, $P e=\delta Q / 2 D_{0}$, is large (here $Q$ is the areal fluid flux at the inlet, $D_{0}$ the molecular diffusion coefficient of the material being filtered in the absence of surrounding particles, and $\delta$ the aspect ratio of the channel). A similarity solution in the boundary layer is obtained in this case. Bowen \& Williams [6] consider a full numerical solution to the continuity, Navier-Stokes and convective-diffusion equations for crossflow ultrafiltration for concentrationdependent viscosity and diffusion coefficient using a Thomas algorithm. Their results show the significant effect various parameters have on concentration polarization and the rate of filtration.

The transition from concentration polarization to deposition (fouling) on the membrane is of great importance to the lifetime of membranes. Bacchin et. al. [7] model 
this by a toggle that changes the equations for the permeate flux and wall concentration depending on which is taking place. This toggle is implemented via a critical concentration: below this critical value, concentration polarization occurs, but once above it, there is sufficiently high concentration for particle deposition to occur as well as concentration polarization (i.e., there is some irreversible solidification as a gel or cake formation). The type of driving phenomena may alter the outcome and the critical flux for the transition between concentration polarization and deposition is linked to the local Péclet number.

In this paper we consider a pressure-driven fluid flow with advection and diffusion of particles in a porous channel. We study the particular case of a dilute suspension of identical and neutrally buoyant particles where we consider a bulk concentration with a specified cross-stream distribution entering the channel. As such, we assume that no deposition of particles on the membrane occurs. We assume a concentration-dependence of the viscosity of the fluid and determine that a constant diffusion coefficient is sufficient. We assume that the flow is steady in time and examine its variations in space arising due to the local particle concentration and leakage flow through the channel walls. We allow fluid (but not particles) to pass through the porous walls so that the walls act as perfect filters, and we do not consider the possibility that particles may block pores when they reach the walls. We retrieve the fluid flow as in [2] as a leading-order solution that does not have a particle concentration dependence, and we determine a higher-order solution that does depend on particle concentration. We also consider the case of a pressure-dependent leakage velocity where the hydrodynamic and osmotic pressures drive the filtration. We are particularly interested in the pressures required for a constant inlet fluid flux due to the concentration-dependent viscosity.

In $\S 2$ we provide a mathematical description of the problem, applying a thin-channel approximation to the governing equations to derive a coupled system of nonlinear partial differential equations (PDEs). We simplify these equations in $\S 3$ by examining the asymptotic limit of a dilute suspension of particles and solve these equations in $\S 4$ with analytic results at leading order and numerical results at the next order. In $\S 5$ we consider the effects of an outer pressure, that is, a pressure outside the channel that affects the transmembrane pressure difference. We have taken order-one Péclet numbers, meaning that the advection and diffusion of particles in the channel are equally important to provide the greatest generality for the particle dynamics in the channel.

\section{Modelling}

We consider a two-dimensional channel of length $L$, with porous walls located at $z= \pm H / 2$, as depicted in Figure 1. Fluid is injected into the channel at $x=0$ at a fixed areal flux, $Q$, and with a given concentration

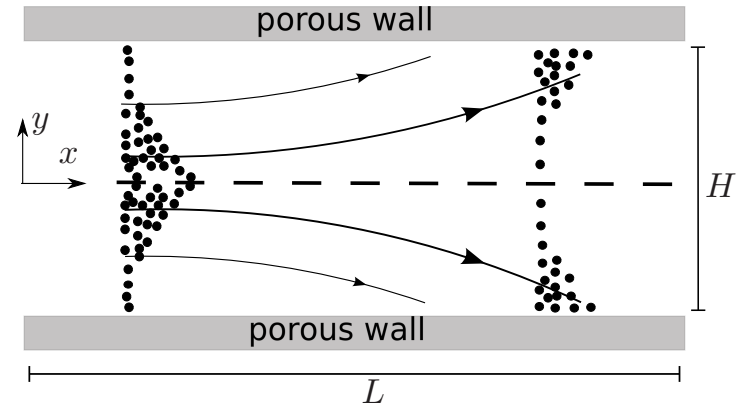

Figure 1: Schematic of a channel with porous walls. The channel has length $L$ and width $H$. The fluid flows from left to right and is allowed to leak out through the channel walls. Particles entrained in the fluid with some distribution at the inlet are advected by the flow and diffuse by Brownian motion, collecting at the channel walls.

distribution of particles. The velocity field within the fluid is $\boldsymbol{u}=(u, v)$, with $u$ the axial component and $v$ the transverse component. In such a configuration the solvent (particle-free) viscosity, $\mu_{0}$, will play a key role in determining the flow profile. We shall assume a small Reynolds number, $R e=\rho_{0} Q / \mu_{0} \ll 1$ (with $\rho_{0}$ the density of the fluid) and hence assume that inertia may be neglected. Furthermore, we shall only consider the steady state of the system.

For convenience, we will define the concentration of particles, $c\left(\mathrm{~mol} / \mathrm{m}^{3}\right)$, in terms of the volume fraction, $\phi$, that is, the volume occupied by the particles. The volume fraction is linearly related to the concentration via

$$
\phi=N_{A} V_{p} c
$$

where $N_{A}$ is Avogadro's number and $V_{p}$ is the volume occupied by a single particle. We suppose that the volume fraction of particles at the inlet, $x=0$, is prescribed by $\phi(x=0, y)=\Phi(y)$ for some function $\Phi$. We wish to understand how the volume fraction, $\phi(x, y)$, changes with distance along the channel as a result of the fluid flow, and how it influences the flow. We shall therefore also assume that the viscosity of the fluid, $\mu$, is dependent on the volume fraction, writing $\mu=\mu(\phi)$, the functional form of which will be prescribed in due course.

\subsection{Governing equations}

Assuming a small Reynolds number, the fluid flow is governed by the steady Stokes equations, representing conservation of mass and momentum of the fluid. We may write these as

$$
\begin{aligned}
\boldsymbol{\nabla} \cdot \boldsymbol{u} & =0, \\
\boldsymbol{\nabla} \cdot\left(\mu(\phi)\left[\boldsymbol{\nabla} \boldsymbol{u}+(\boldsymbol{\nabla} \boldsymbol{u})^{T}\right]\right) & =\boldsymbol{\nabla} p,
\end{aligned}
$$

where $p$ is the hydrodynamic fluid pressure.

The flow advects the particles down the channel, but they also diffuse within the channel. The particle volume 
fraction is therefore governed by the steady advectiondiffusion equation,

$$
\boldsymbol{u} \cdot \boldsymbol{\nabla} \phi=\boldsymbol{\nabla} \cdot(D(\phi) \boldsymbol{\nabla} \phi) .
$$

Here $D(\phi)$ is the particle diffusivity, which will in principle depend on volume fraction.

\subsection{Boundary conditions}

We now consider the boundary conditions to which the governing equations (2) and (3) are subjected. We assume that the flow and particle distribution are symmetric about the axis of the channel, that is,

$$
\frac{\partial \phi}{\partial y}=\frac{\partial u}{\partial y}=v=0 \quad \text { on } y=0
$$

Henceforth we shall consider only the behaviour in half channel $0 \leq y \leq H / 2$. We consider the two leakage flows through the porous channel walls, $\mathcal{V}:=v(x, H / 2)$, that are most often found in practical applications. First we consider the case when $\mathcal{V}$ is a constant, say $\mathcal{V}^{*}$. In the absence of significant osmotic effects, this type of leakage flow has been achieved by having a flow of fluid (recirculated filtered fluid) outside the channel so that the pressure difference across the membrane is constant along the wall [8]. Secondly we consider the case where $\mathcal{V}$ is proportional to the net driving force (i.e., the transmembrane pressure less the difference in osmotic pressure). At the wall, we therefore have one of the two possible boundary conditions for the transverse velocity,

$$
v(x, H / 2)=\mathcal{V}=\left\{\begin{array}{l}
\mathcal{V}^{*}=\text { constant } \\
\kappa(\Delta p-\Delta \pi),
\end{array}\right.
$$

respectively, where $\kappa$ is a constant of proportionality that is related to the solvent viscosity and the permeability of the porous wall and its thickness ( $c f$. Darcy's law). Here the term $\Delta p=p(x)-p_{\text {outer }}$ is the hydrodynamic pressure difference across the membrane, which is measured relative to the pressure outside the channel, $p_{\text {outer }}$ (i.e., the reference pressure). Similarly, $\Delta \pi=\pi-\pi_{\text {outer }}$ where $\pi$ is the osmotic pressure due to particles in the channel and $\pi_{\text {outer }}$ is that due to particles outside the channel. Here, we take $\pi_{\text {outer }}=0$ since we assume complete rejection of particles at the channel wall. The osmotic pressure difference across the porous wall is, in general, a function of the volume fraction of particles at the surface of the porous wall

$$
\Delta \pi=\Delta \pi\left[\phi\left(x, y=\frac{H}{2}\right)\right]
$$

since we assume that there are no particles on the other side of the membrane.

In general, at a porous wall there is a tangential slip velocity, whose magnitude is determined by a Neumann boundary condition such as that given by Beavers and
Joseph [9]. However, it has been found that this slip is not significant for a wide range of membranes [10], and so here, for simplicity, we shall assume a no-slip boundary condition

$$
u(x, H / 2)=0,
$$

as also adopted by Bowen \& Williams [6].

Since particles are rejected by the membrane but fluid may pass through, we use a no-flux boundary condition for the particles at the channel walls. This may be written as $[4,7]$

$$
v \phi-D \frac{\partial \phi}{\partial y}=0 \quad \text { on } y=H / 2 .
$$

Two final conditions close the system. At the inlet we impose a constant fluid flux condition

$$
\int_{-H / 2}^{H / 2} u \mathrm{~d} y=2 \int_{0}^{H / 2} u \mathrm{~d} y=Q \quad \text { at } x=0,
$$

where $Q$ is the constant areal flux. At the outlet we require that the pressure be constant; without loss of generality we may set this outlet pressure to to zero, i.e.,

$$
p=0 \quad \text { at } \quad x=L,
$$

but note that this may differ from the pressure outside the channel and so $p_{\text {outer }}$ may not necessarily be zero.

\subsection{Thin-channel approximation}

We suppose that the channel is thin so that the aspect ratio $H / 2 L=\delta \ll 1$. We exploit the smallness of $\delta$, nondimensionalizing the system by letting

$$
\begin{aligned}
& x=L \hat{x}, \quad y=\delta L \hat{y}, \quad u=\frac{Q}{H} \hat{u}, \\
& v=\delta \frac{Q}{H} \hat{v}, \quad \mu=\mu_{0} \hat{\mu}, \quad \quad p=\frac{Q \mu_{0}}{\delta^{2} H L} \hat{p}, \\
& \kappa=\frac{\delta^{3} L}{\mu_{0}} \hat{\kappa}, \quad \pi=\frac{Q \mu_{0}}{\delta^{2} H L} \hat{\pi}
\end{aligned}
$$

in which the dimensionless variables appear with a hat and $\mu_{0}$ is the viscosity of the solvent in the absence of particles. Substituting the non-dimensionalization (11) into equations (2) and (3) and retaining only leading-order terms in $\delta$ provides the equations for the volume fraction in a thin-channel flow with a concentration-dependent viscosity (dropping the hats for convenience),

$$
\begin{aligned}
\frac{\partial u}{\partial x}+\frac{\partial v}{\partial y} & =0 \\
\frac{\partial}{\partial y}\left(\mu(\phi) \frac{\partial u}{\partial y}\right) & =\frac{\partial p}{\partial x} \\
0 & =\frac{\partial p}{\partial y} \\
P e\left(u \frac{\partial \phi}{\partial x}+v \frac{\partial \phi}{\partial y}\right) & =\frac{\partial}{\partial y}\left(D(\phi) \frac{\partial \phi}{\partial y}\right) .
\end{aligned}
$$


Here $P e$ is the (reduced) Péclet number,

$$
P e=\frac{\delta}{2} \frac{Q}{D_{0}},
$$

with $D_{0}=D(0)$ the molecular diffusion coefficient, that is, the diffusion coefficient in the absence of the effects of surrounding particles. This is given by the StokesEinstein relation. The Péclet number measures the rate of advection of particles down the channel compared with the diffusion across the channel. As discussed earlier, the viscosity $\mu(\phi)$ is assumed to be a known function. We note that (12c) implies that the hydrodynamic pressure is, to leading order, a function of $x$ only, i.e., $p=p(x)$. Note also that equation (12d) indicates that there is no axial particle diffusion present at leading order in $\delta$.

The dimensionless boundary conditions, to leading order in $\delta$, to be used in determining the solution to (12) are as follows (again dropping hats for convenience),

$$
\begin{aligned}
& \frac{\partial \phi}{\partial y}=\frac{\partial u}{\partial y}=v=0 \quad \text { on } y=0, \\
& v(x, 1)=\mathcal{V}=\left\{\begin{array}{l}
\mathcal{V}^{*}, \\
\kappa\left[p-p_{\text {outer }}-\Delta \pi\right],
\end{array}\right. \\
& u(x, 1)=0, \\
& \text { Pe } v \phi-\frac{\partial \phi}{\partial y}=0 \quad \text { on } y=1 \text {, } \\
& \int_{0}^{1} u \mathrm{~d} y=1 \quad \text { at } x=0, \\
& p=0 \quad \text { at } x=1 \text {. }
\end{aligned}
$$

Finally, we specify the volume fraction profile at the inlet

$$
\phi(0, y)=\Phi(y),
$$

for some $\Phi(y)$. Two natural types of injection that should be compared are (i) uniform injection across $0 \leq y \leq 1$ and (ii) point injection. To capture both of these we consider a normalized particle distribution at the inlet that is Gaussian in nature, of the form

$$
\phi(0, y)=\Phi(y)=\frac{\exp \left(\frac{-y^{2}}{2 \sigma^{2}}\right)}{\sqrt{\frac{\pi}{2}} \sigma \operatorname{erf}\left(\frac{1}{\sqrt{2} \sigma}\right)} .
$$

Here $\sigma$ is a constant that reflects the width of the distribution. The uniform inlet volume fraction arises in the limit $\sigma \rightarrow \infty$, whilst point injection at the centre $y=0$ corresponds to $\sigma \rightarrow 0$; intermediate values of $\sigma$ give different pulse widths.

Equations (12) subject to boundary conditions (14) and inlet condition (15) define our problem mathematically.

\subsection{Model solution}

Our aim is to determine the change in particle volume fraction, $\phi$, as we move down the channel, i.e., the variation with $y$ of $\phi(x, y)$ as $x$ increases. Integrating the momentum equation (12b), and making use of the symmetry and no-slip conditions $(14 \mathrm{a}, 14 \mathrm{c})$ gives

$$
u(x, y)=-\frac{\mathrm{d} p}{\mathrm{~d} x} \int_{y}^{1} \frac{\tilde{y}}{\mu(\phi(x, \tilde{y}))} \mathrm{d} \tilde{y} .
$$

Substituting (17) into the continuity equation (12a), integrating and applying (14a) gives

$$
\begin{aligned}
v(x, y)=\frac{\partial}{\partial x}\left\{\frac { \mathrm { d } p } { \mathrm { d } x } \left(y \int_{y}^{1} \frac{\tilde{y}}{\mu(\phi(x, \tilde{y}))} \mathrm{d} \tilde{y}\right.\right. \\
\left.\left.+\int_{0}^{y} \frac{\tilde{y}^{2}}{\mu(\phi(x, \tilde{y}))} \mathrm{d} \tilde{y}\right)\right\},
\end{aligned}
$$

where we have taken the $x$-derivative outside the integration, and used integration by parts to simplify a double integral. The transverse fluid velocity at the channel wall, $\mathcal{V}$, is then given by

$$
v(x, 1)=\mathcal{V}=\frac{\partial}{\partial x}\left\{\frac{\mathrm{d} p}{\mathrm{~d} x}\left(\int_{0}^{1} \frac{\tilde{y}^{2}}{\mu(\phi(x, \tilde{y}))} \mathrm{d} \tilde{y}\right)\right\} .
$$

Treating $\mathcal{V}$ as given by the two behaviours of the leakage flow of interest in (14b), and expanding the derivative in equation (19), we have an ordinary differential equation (ODE) for the hydrodynamic pressure, $p(x)$, of the form

$$
\frac{\mathrm{d}^{2} p}{\mathrm{~d} x^{2}}-\frac{B(x)}{A(x)} \frac{\mathrm{d} p}{\mathrm{~d} x}-\frac{1}{A(x)} \mathcal{V}=0
$$

where

$$
\begin{aligned}
A(x) & =\int_{0}^{1} \frac{\tilde{y}^{2}}{\mu(\phi(x, \tilde{y}))} \mathrm{d} \tilde{y} \\
B(x) & =\int_{0}^{1} \frac{\tilde{y}^{2}}{\mu(\phi(x, \tilde{y}))^{2}} \frac{\partial \mu(\phi(x, \tilde{y}))}{\partial x} \mathrm{~d} \tilde{y} .
\end{aligned}
$$

Equations (17) and (18) give the axial and transverse velocities in terms of the volume fraction, $\phi$, and hydrodynamic pressure, $p$. These may be substituted into the advection-diffusion equation (12d) and, with the ODE for the hydrodynamic pressure (20), they provide two coupled integro-differential equations for $\phi$ and $p$, which are difficult to solve numerically. However, in the next section we are able to make further analytical progress by exploring the limit of a dilute suspension, $\phi \ll 1$.

\section{Asymptotics for a Dilute Suspension}

The coupled nonlinear system of equations (12) can be simplified by considering a dilute suspension of particles,

$$
\phi(x, y)=\epsilon \phi_{1}(x, y)
$$


with $\epsilon \ll 1$ and $\phi_{1}$ assumed to be $\mathcal{O}(1)$.

The osmotic pressure difference across the porous wall, in the dilute limit, is a linear function of the particle volume fraction [11]

$$
\Delta \pi=\epsilon \pi_{0} \phi_{1}(x, y=1)+\mathcal{O}\left(\epsilon^{2}\right),
$$

where $\pi_{0}$ is a reference osmotic pressure. In the dilute limit, the leading-order effect of volume fraction on viscosity is given by the Einstein viscosity [12]

$$
\mu(\phi)=1+\frac{5}{2} \epsilon \phi_{1}+\mathcal{O}\left(\epsilon^{2}\right) .
$$

Finally, the asymptotic expression for the effective diffusion coefficient, $D(\phi)$, for a dilute mono-disperse suspension is given by [13]

$$
D(\phi)=1+\epsilon \chi \phi_{1},
$$

where the constant $\chi$ is a virial coefficient, a type-specific constant accounting for particle-particle interactions (see $[13,14]$ for tabulated values from the literature).

We exploit the dilute approximation by expanding the hydrodynamic pressure and velocity components as

$$
\begin{aligned}
p(x) & =p_{0}(x)+\epsilon p_{1}(x)+\mathcal{O}\left(\epsilon^{2}\right), \\
u(x, y) & =u_{0}(x, y)+\epsilon u_{1}(x, y)+\mathcal{O}\left(\epsilon^{2}\right), \\
v(x, y) & =v_{0}(x, y)+\epsilon v_{1}(x, y)+\mathcal{O}\left(\epsilon^{2}\right) .
\end{aligned}
$$

Substituting these expressions into the advection-diffusion equation for $\phi,(12 \mathrm{~d})$, we find that the lowest-order terms are at $\mathcal{O}(\epsilon)$, and so this forms an equation for $\phi_{1}$ in terms of the leading-order velocities $u_{0}$ and $v_{0}$ :

$$
P e\left(u_{0} \frac{\partial \phi_{1}}{\partial x}+v_{0} \frac{\partial \phi_{1}}{\partial y}\right)=\frac{\partial^{2} \phi_{1}}{\partial y^{2}} .
$$

We note that the Stokes-Einstein diffusivity, $D_{0}$, enters through the Péclet number and also that the concentrationdependent terms in the diffusivity only appear at order $\epsilon^{2}$, which are neglected. Hence, we need only consider a constant diffusivity, $D_{0}$, here. The symmetry and no-flux boundary conditions from (14a) and (14d) read

$$
\begin{aligned}
\frac{\partial \phi_{1}}{\partial y}=0 & \text { on } y=0, \\
P e \mathcal{V}_{0} \phi_{1}-\frac{\partial \phi_{1}}{\partial y}=0 & \text { on } y=1,
\end{aligned}
$$

where $\mathcal{V}_{0}=v_{0}(x, 1)$ is the leading-order leakage flow at the porous channel walls. The inlet condition is

$$
\phi_{1}(0, y)=\Phi_{1}(y),
$$

for a normalized Gaussian function $\Phi(y)$ of the form given by equation (16).
The expression for the axial velocity given by (17) may be expanded in powers of $\epsilon$, using the viscosity and pressure expansions (24) and (26a), to give

$$
\begin{aligned}
& u(x, y)=\frac{\mathrm{d} p_{0}}{\mathrm{~d} x} \frac{\left(y^{2}-1\right)}{2} \\
& +\epsilon\left(\frac{\mathrm{d} p_{1}}{\mathrm{~d} x} \frac{\left(y^{2}-1\right)}{2}+\frac{\mathrm{d} p_{0}}{\mathrm{~d} x} \int_{y}^{1} \frac{5}{2} \tilde{y} \phi_{1}(x, \tilde{y}) \mathrm{d} \tilde{y}\right)+\mathcal{O}\left(\epsilon^{2}\right) .
\end{aligned}
$$

Similarly for the transverse velocity, given by (18), we find

$$
\begin{aligned}
v(x, y)= & \frac{\mathrm{d}^{2} p_{0}}{\mathrm{~d} x^{2}} \frac{\left(3 y-y^{3}\right)}{6} \\
+\epsilon \frac{\partial}{\partial x}\left\{\frac{\mathrm{d} p_{1}}{\mathrm{~d} x}\right. & \frac{\left(3 y-y^{3}\right)}{6}-\frac{\mathrm{d} p_{0}}{\mathrm{~d} x}\left[y \int_{y}^{1} \frac{5}{2} \tilde{y} \phi_{1}(x, \tilde{y}) \mathrm{d} \tilde{y}\right. \\
& \left.\left.+\int_{0}^{y} \frac{5}{2} \tilde{y}^{2} \phi_{1}(x, \tilde{y}) \mathrm{d} \tilde{y}\right]\right\}+\mathcal{O}\left(\epsilon^{2}\right) .
\end{aligned}
$$

Equations (30) and (31) determine the leading-order and order- $\epsilon$ components of the velocities $u$ and $v$. As expected in the dilute limit, the presence of particles does not affect the leading-order problem; their effect is only felt at the next order.

The two cases for the boundary condition for $v$ at $y=1(14 \mathrm{~b})$ take the form $\mathcal{V}=\mathcal{V}_{0}+\epsilon \mathcal{V}_{1}$ where

$$
\mathcal{V}=\mathcal{V}_{0}+\epsilon \mathcal{V}_{1}=\left\{\begin{array}{l}
\mathcal{V}^{*}, \\
\kappa\left(p_{0}-p_{\text {outer }}^{0}\right) \\
\quad+\epsilon \kappa\left[p_{1}-p_{\text {outer }}^{1}-\pi_{0} \phi_{1}(x, y=1)\right]
\end{array}\right.
$$

Thus there is no $\mathcal{O}(\epsilon)$ correction to the leakage flow for the case of constant outflow, i.e., $\mathcal{V}_{1}=0$ in this case. However, for the pressure-dependent leakage flow, the leading-order outflow is proportional to the leading-order pressure difference across the membrane, and the order- $\epsilon$ outflow is related to the order- $\epsilon$ hydrodynamic pressure and the osmotic pressure.

\section{Results}

\subsection{Leading-order velocities}

Examining the $\mathcal{O}(1)$ velocity terms in the expansions (30) and (31), we find the leading-order velocity components $\boldsymbol{u}_{0}=\left(u_{0}, v_{0}\right)$,

$$
\begin{aligned}
& u_{0}(x, y)=\frac{\mathrm{d} p_{0}}{\mathrm{~d} x} \frac{\left(y^{2}-1\right)}{2}, \\
& v_{0}(x, y)=\frac{\mathrm{d}^{2} p_{0}}{\mathrm{~d} x^{2}} \frac{\left(3 y-y^{3}\right)}{6} .
\end{aligned}
$$

Note that the axial velocity, $u_{0}$, depends quadratically on the transverse coordinate, $y$, and the transverse velocity, $v_{0}$, depends cubically on $y$. Similar channel velocities may be found in the literature (e.g., see Probstein [2] 
for the case of a constant leakage outflow). If the hydrodynamic pressure gradient, $\mathrm{d} p_{0} / \mathrm{d} x$, were constant, the leading-order velocities (33) would correspond precisely to Poiseuille flow. However, here we have the added interest that $\mathrm{d} p_{0} / \mathrm{d} x$ may be a function of $x$. This means that, while the profile remains parabolic, its magnitude may vary due to the spatially varying hydrodynamic pressure, $p_{0}$. This variation must be determined case by case by considering the leading-order flow through the channel wall $\mathcal{V}_{0}$

$$
v_{0}(x, 1)=\mathcal{V}_{0}=\frac{1}{3} \frac{\mathrm{d}^{2} p_{0}}{\mathrm{~d} x^{2}}=\left\{\begin{array}{l}
\mathcal{V}^{*}, \\
\kappa p_{0} .
\end{array}\right.
$$

We note that, in solving for the leading-order hydrodynamic pressure $p_{0}$ from the ODE in (34), we use the constraints of constant flux at the channel inlet (14e) and zero pressure at the outlet (14f), which, for the leadingorder problem, read as

$$
\begin{aligned}
\int_{0}^{1} u_{0} \mathrm{~d} y & =1, & & \text { at } x=0, \\
p_{0} & =0, & \text { at } x & =1 .
\end{aligned}
$$

In the following we consider each of these two cases, in turn.

\subsubsection{Case 1: Constant leakage}

In the case of constant flow through the porous walls, $\mathcal{V}_{0} \equiv \mathcal{V}^{*}=$ constant, the ODE for the leading-order hydrodynamic pressure (34) with conditions (35) has solution

$$
p_{0}=3(1-x)\left[1-\frac{1}{2} \mathcal{V}^{*}(x+1)\right] \text {. }
$$

This function decreases with $\mathcal{V}^{*}$ for all $x$, i.e., the greater the leakage flow the lower the required hydrodynamic pressure to maintain a constant flux. Hence, for constant flow though the walls, the pressure gradient changes linearly with distance down the channel. The leading-order flow then reads

$$
\begin{aligned}
\boldsymbol{u}_{0} & =\left(u_{0}, v_{0}\right), \\
& =\left(\frac{3}{2}\left(1-y^{2}\right)-\frac{3}{2} \mathcal{V}^{*} x\left(1-y^{2}\right), \frac{1}{2} \mathcal{V}^{*}\left(3 y-y^{3}\right)\right),
\end{aligned}
$$

from (33). The leading-order axial velocity $u_{0}=u_{0}(x, y)$ and the transverse velocity $v_{0}=v_{0}(y)$ are dependent on the magnitude of the flow through the walls. Figure 2 shows the leading-order velocity profiles for $\mathcal{V}^{*}=0.25$. The leading-order axial velocity has a parabolic profile but its magnitude decreases linearly down the channel, corresponding to a retardation of the fluid due to the leakage through the channel walls. The leading-order transverse velocity has a cubic profile as a result of the flow towards the channel walls, which does not vary with axial position. In the case of no leakage flow (impermeable walls), $\mathcal{V}^{*}=0$, and (37) reduces simply to Poiseuille flow.
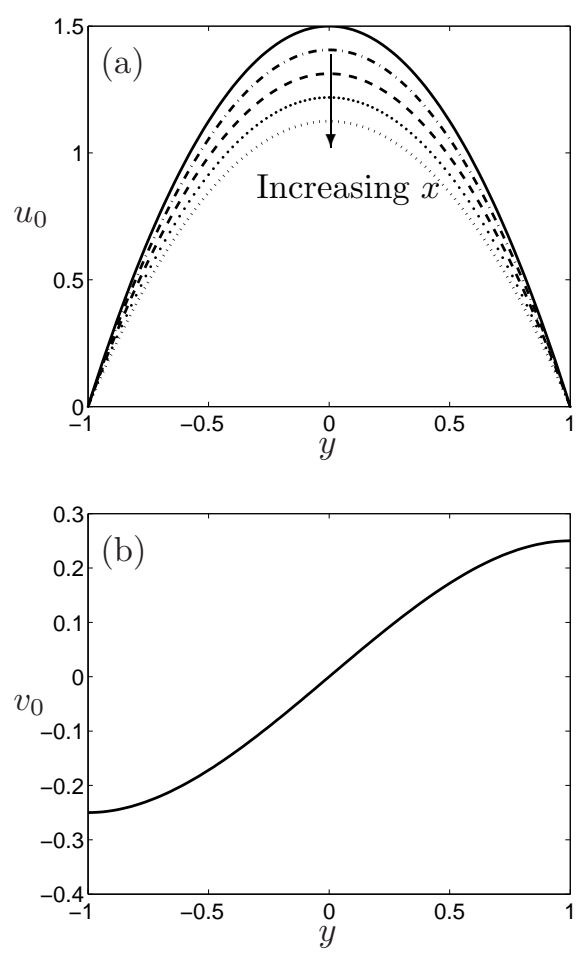

Figure 2: Profiles of the leading-order velocities (37) with constant leakage flow $\mathcal{V}^{*}=0.25$ through the channel walls. (a) Axial velocity, $u_{0}$, at $x=0$ (solid), $x=0.25$ (dot-dashed), $x=0.5$ (dashed), $x=0.75$ (dotted) and $x=1$ (skinny-dotted), and (b) Transverse velocity, $v_{0}$. We observe that $v_{0}=v_{0}(y)$ in the channel.

There is a maximum allowable constant leakage flow, $\mathcal{V}_{\max }^{*}$, for which there is a positive net axial flow at the end of the channel; in this limiting case, all the fluid injected at the inlet passes through the porous walls. Exceeding this leakage flow velocity results in back-flow from the channel outlet into the channel. The value of $\mathcal{V}_{\max }^{*}$ is obtained by equating the influx of fluid with the flux through the channel walls, given by integrating the velocity $v_{0}(37)$ along the channel wall at $y=1$, resulting in a maximum leakage flow of $\mathcal{V}_{\max }^{*}=1$. Such a dead-end flow occurs in direct-flow filtration [15].

\subsubsection{Case 2: Pressure-dependent leakage}

We now determine leading-order and order- $\epsilon$ solutions for the velocities, pressure and volume fraction for a pressure-dependent leakage flow. For simplicity we assume zero pressure outside the channel, $p_{\text {outer }} \equiv 0$. This corresponds to the common set-up in which the hydrodynamic outlet pressure and pressure outside the channel walls are equal. In $\$ 5$ we relax this assumption to explore the effect of a non-zero $p_{\text {outer }}$.

When the leakage flow through the wall is proportional to the hydrodynamic pressure, $p$, and osmotic pressure, $\pi$, then at leading order only the hydrodynamic pressure is significant, $\mathcal{V}_{0}=\kappa p_{0}$, as seen from equation (32). The leading-order transverse flow at $y=1$ (34) gives an ODE 

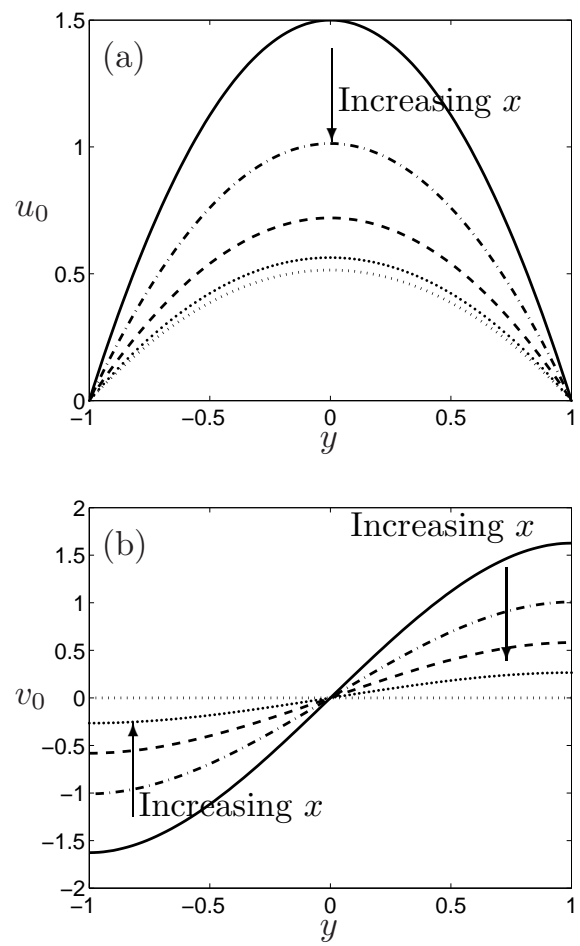

Figure 3: Profiles of the leading-order velocities for pressuredependent leakage flow with $\kappa=1$ so that $\mathcal{V}_{0}=p_{0}$ : (a) axial velocity, $u_{0}$, and (b) transverse velocity, $v_{0}$. In each, we show the profiles at $x=0$ (solid), $x=0.25$ (dot-dashed), $x=0.5$ (dashed), $x=0.75$ (dotted) and $x=1$ (skinny-dotted).

for the leading-order hydrodynamic pressure

$$
\frac{1}{3} \frac{\mathrm{d}^{2} p_{0}}{\mathrm{~d} x^{2}}=\kappa p_{0}
$$

This ODE, subject to the boundary conditions (35), has the solution

$$
p_{0}=\sqrt{\frac{3}{\kappa}} \operatorname{sech} \sqrt{3 \kappa} \sinh [\sqrt{3 \kappa}(1-x)] .
$$

From this equation, we see that the pressure, $p_{0}$, decreases with $\kappa$ for all $x$ and, as is the case for uniform leakage, the greater the leakage through the channel walls, the lower the required hydrodynamic pressure to achieve a constant influx. Substituting (39) into (33) determines the leadingorder velocity field, $\left(u_{0}, v_{0}\right)$. We note that, as $\kappa \rightarrow 0$ (in the limit of impermeable walls), $p_{0} \rightarrow 3(1-x)$, and we recover Poiseuille flow, as expected. The components of the leading-order velocity field are shown in Figure 3 for $\kappa=1$. We observe that the leading-order axial velocity $u_{0}$ retains its parabolic profile along the channel though the amplitude decreases exponentially, because of the leakage of fluid through the channel walls. The leading-order transverse fluid velocity $v_{0}$, which has a cubic profile about $y=0$, also decreases in magnitude along the channel. This indicates the tendency of the fluid to move towards the porous walls of the channel but with an exponentially decaying rate along the channel.

\subsection{Numerical results for volume fraction}

Having seen that the leading-order velocity fields and pressure gradient can be determined analytically in the two cases of interest, we now turn our attention to determining the volume fraction profile, $\phi_{1}$. Recall that the advection-diffusion equation (27), with $u_{0}$ and $v_{0}$ given by our previous analysis, must be solved subject to the boundary conditions (28) and the Gaussian inlet particle distribution (29). We note that this initial condition does not satisfy the no-flux boundary condition (14d) and so there is a small transient over which this relaxes to a configuration that satisfies the boundary conditions. However, we choose to employ this inlet condition as it provides a simple expression that elucidates the effect of a non-uniform particle volume fraction distribution, and we do not expect the behaviour in the small transient to have an effect on the global system dynamics. We solve this system numerically, implementing a scheme in MATLAB (see Appendix for details). As an illustrative example, Figure 4 depicts how an initial distribution in the channel develops downstream of the injection point, with $\sigma^{2}=0.05, P e=3$, for the cases of: no leakage at the wall; constant leakage; and pressure-dependent leakage (with $\kappa=1$ ).

For the case of an impermeable wall, $\mathcal{V}^{*}=0$, we observe that the initial distribution spreads until it is essentially uniform across the width of the channel (Figure $4(\mathrm{a}))$. With leakage flow through the porous wall, particles still diffuse away from the centre-line but now collect near the wall. This build-up of particles at the porous walls is known as concentration polarization [1]. In particular, for the case of constant leakage, the volume fraction of particles at the wall increases monotonically along the length of the channel (Figure 4(b)). When leakage is proportional to the pressure, the volume fraction at the walls is greatest about half way along the channel, as in Figure 4(c). This is as a result of the transverse flow, which becomes smaller near the end of the channel after which point diffusion acts to move particles away from the wall.

\subsection{Order- $\epsilon$ velocities}

Having computed the leading-order velocity components, $u_{0}$ and $v_{0}$, and volume-fraction distribution $\phi_{1}$, we are now in a position to compute the perturbation to the fluid flow caused by the presence of particles, i.e., the $\mathcal{O}(\epsilon)$ corrections to the fluid velocity. From the axial and transverse velocities, (30) and (31) respectively, we have

$$
\begin{gathered}
u_{1}(x, y)=\frac{\mathrm{d} p_{1}}{\mathrm{~d} x} \frac{\left(y^{2}-1\right)}{2}+\frac{\mathrm{d} p_{0}}{\mathrm{~d} x} \int_{y}^{1} \frac{5}{2} \tilde{y} \phi_{1}(x, \tilde{y}) \mathrm{d} \tilde{y}, \\
v_{1}(x, y)=\frac{\partial}{\partial x}\left\{\frac{\mathrm{d} p_{1}}{\mathrm{~d} x} \frac{\left(3 y-y^{3}\right)}{6}-\frac{\mathrm{d} p_{0}}{\mathrm{~d} x}\left[y \int_{y}^{1} \frac{5}{2} \tilde{y} \phi_{1}(x, \tilde{y}) \mathrm{d} \tilde{y}\right.\right. \\
\left.\left.+\int_{0}^{y} \frac{5}{2} \tilde{y}^{2} \phi_{1}(x, \tilde{y}) \mathrm{d} \tilde{y}\right]\right\} .
\end{gathered}
$$




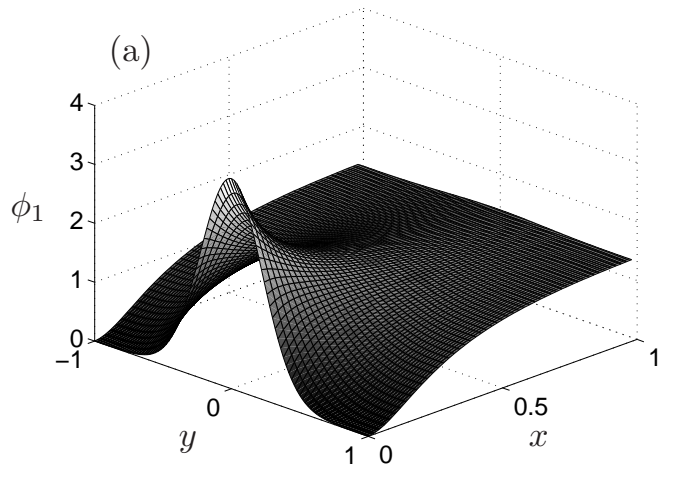

(b)

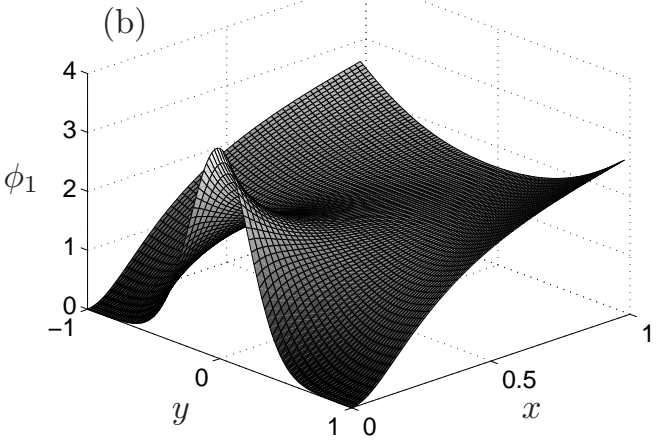

(c)

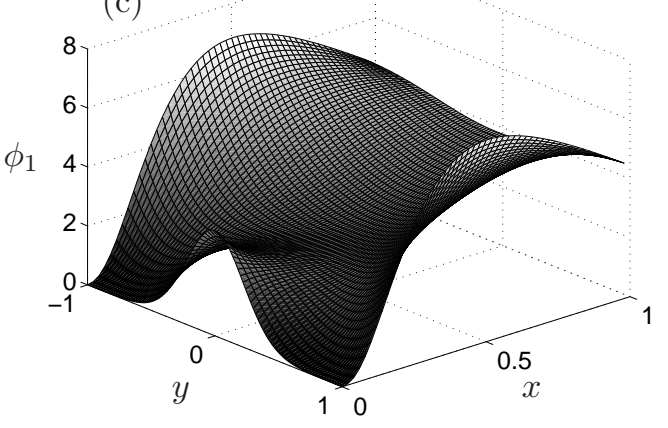

Figure 4: Profiles of the particle volume fraction $\phi_{1}$ in the channel given by the solution to (27) with velocities (33). (a) No leakage $\mathcal{V}^{*}=0, \phi_{1}$ tends to a uniform state in the channel, (b) constant leakage $\mathcal{V}^{*}=0.25$, pressure given by (36), and (c) pressuredependent leakage with $p_{0}$ given by (39). In (b) and (c) leakage flow results in particles collecting at the boundaries. In all computations, we take $P e=3, \sigma^{2}=0.05$, and for (c), $\kappa=1$.
We note that a perturbation to the velocity, $u_{1}$, in principle affects the flux of fluid into the channel. However, the condition of constant fluid influx (14e), considering (35a), demands that,

$$
\int_{0}^{1} u_{1}(0, y) \mathrm{d} y=0 .
$$

To ensure that this condition is satisfied we must impose a perturbation to the hydrodynamic pressure at the inlet $x=0$, i.e., $p_{1}(0)=\mathcal{P} \neq 0$. The value of $\mathcal{P}$ indicates how much harder we must push the liquid in the presence of particles to obtain the same fluid influx as would be obtained for a configuration in the absence of particles. The hydrodynamic pressure perturbation, $p_{1}$, may be found from the perturbed leakage $\mathcal{V}_{1}$ out of the channel wall at $y=1$,

$$
\frac{\partial}{\partial x}\left\{\frac{1}{3} \frac{\mathrm{d} p_{1}}{\mathrm{~d} x}-\frac{\mathrm{d} p_{0}}{\mathrm{~d} x} \int_{0}^{1} \frac{5}{2} \tilde{y}^{2} \phi_{1}(x, \tilde{y}) \mathrm{d} \tilde{y}\right\}=\mathcal{V}_{1},
$$

using the transverse velocity (31). Here $\mathcal{V}_{1}=0$ for the case of constant leakage flow through the channel walls and $\mathcal{V}_{1}=\kappa\left(p_{1}-\pi_{0} \phi_{1}\right)$ when the leakage flow is pressuredependent, see equation (32). For each case we may substitute for $p_{0}$ in equation (42) and solve subject to

$$
p_{1}(0)=\mathcal{P}, \quad p_{1}(1)=0 .
$$

We then use the flux condition (41) to find $\mathcal{P}$.

By considering the initial volume fraction distribution given by (16) we may determine the dependence of $\mathcal{P}$ on the width of the distribution, $\sigma$. This gives us insight into the pressures required to transport a fixed flux of fluid containing a given distribution of particles through the channel for the two distinct cases of leakage through the channel walls, $\mathcal{V}_{1}$.

\subsubsection{Case 1: Constant leakage}

When there is constant flow through the boundary, $\mathcal{V}=\mathcal{V}^{*}$, and so, at order $\epsilon, \mathcal{V}_{1}=0$. In this case, the ODE (42) to determine the order- $\epsilon$ pressure, $p_{1}$, becomes

$$
\frac{1}{3} \frac{\mathrm{d}^{2} p_{1}}{\mathrm{~d} x^{2}}-\frac{\partial}{\partial x}\left\{\frac{\mathrm{d} p_{0}}{\mathrm{~d} x} \int_{0}^{1} \frac{5}{2} \tilde{y}^{2} \phi_{1}(x, \tilde{y}) \mathrm{d} \tilde{y}\right\}=0,
$$

which has solution

$$
\begin{aligned}
p_{1}= & \mathcal{P}(1-x)+3 \int_{0}^{x}\left[\frac{\mathrm{d} p_{0}(\tilde{x})}{\mathrm{d} \tilde{x}} \int_{0}^{1} \frac{5}{2} \tilde{y}^{2} \phi_{1}(\tilde{x}, \tilde{y}) \mathrm{d} \tilde{y}\right] \mathrm{d} \tilde{x} \\
& -3 x \int_{0}^{1}\left[\frac{\mathrm{d} p_{0}(\tilde{x})}{\mathrm{d} \tilde{x}} \int_{0}^{1} \frac{5}{2} \tilde{y}^{2} \phi_{1}(\tilde{x}, \tilde{y}) \mathrm{d} \tilde{y}\right] \mathrm{d} \tilde{x},
\end{aligned}
$$

where we have applied the boundary conditions (43). Upon imposing the flux condition (41), we see that the input pressure perturbation, $\mathcal{P}$, is related to the particle volume 
fraction through

$$
\begin{aligned}
\mathcal{P}= & 3 \frac{\mathrm{d} p_{0}(0)}{\mathrm{d} x} \int_{0}^{1} \frac{5}{2} \tilde{y}^{2} \phi_{1}(0, \tilde{y}) \mathrm{d} \tilde{y} \\
& -3 \int_{0}^{1}\left[\frac{\mathrm{d} p_{0}(\tilde{x})}{\mathrm{d} \tilde{x}} \int_{0}^{1} \frac{5}{2} \tilde{y}^{2} \phi_{1}(\tilde{x}, \tilde{y}) \mathrm{d} \tilde{y}\right] \mathrm{d} \tilde{x} \\
& -3 \int_{0}^{1} \frac{\mathrm{d} p_{0}(0)}{\mathrm{d} x} \int_{\tilde{y}}^{1} \frac{5}{2} y^{\prime} \phi_{1}\left(0, y^{\prime}\right) \mathrm{d} y^{\prime} \mathrm{d} \tilde{y} .
\end{aligned}
$$

We note that this relationship is non-local, depending on the behaviour of the particles along the entire length of the channel. Substituting for $\mathcal{P}$ into (45) provides the pressure perturbation, $p_{1}$, and, along with $\phi_{1}$, allows us to calculate the perturbations to the velocity field $u_{1}$ and $v_{1}$ using the order- $\epsilon$ velocities (40).

In Figure 5(a) we illustrate the pressure for the profile $\phi_{1}$ with $\sigma^{2}=0.05$ and $P e=3$, as shown in Figure 4(b). As we expect, the pressure perturbation is positive for all $x$ : the hydrodynamic pressure in the channel required to maintain the same fluid influx as the case in which no particles are present is increased as a result of the increase in viscosity. The perturbation to the axial velocity, $u_{1}$, is shown in Figure 5(b). Recall that the leading-order axial velocity here is a Poiseuille profile, decreasing in magnitude as we move down the channel. We see that, in regions of high particle volume fraction, $u_{1}$ is negative and so the total axial velocity, $u$, is decreased by the presence of particles, while in regions of lower particle volume fraction $u_{1}$ is positive and so the total axial velocity is increased. By conservation of mass, (12a), a perturbation in the axial velocity results in a perturbation in the transverse velocity, $v$. Since there is an accumulation of particles near the walls, i.e., the region of high volume fraction moves to the walls, the location of the position of maximum retardation in the transverse direction caused by the perturbation to the flow moves towards the walls as we move down the channel (Figure 5(b)). Hence, while the perturbed transverse velocity, $v_{1}$ (40b), initially increases, since there is no perturbed leakage through the walls, i.e., $v_{1}(x, 1)=0$, the fluid must ultimately be transported back towards the centre of the channel; this is signified by a change in sign of $v_{1}$ further along the channel, as seen in Figure 5(c).

The relationship between the pressure perturbation at the inlet, $\mathcal{P}$, and the width of the particle distribution, $\sigma$, for various constant leakage velocities, $\mathcal{V}^{*}$, is shown in Figure 6 . Since the addition of particles increases the viscosity of the fluid, and the hydrodynamic pressure gradient is related to the viscosity through the momentum equation $(12 \mathrm{~b})$, we expect that a greater hydrodynamic pressure will be required to maintain a constant influx. However, we find that that there is a critical value of $\sigma$ that maximizes the additional pressure, $\mathcal{P}$. The critical value of the distribution width, $\sigma_{\text {pess }}$, decreases linearly with leakage flow, $\mathcal{V}^{*}$, as in Figure $7($ a). This means, surprisingly, that there is an inlet particle distribution width that requires the largest additional (perturbed) pressure. The value of this pessimal pressure perturbation increases
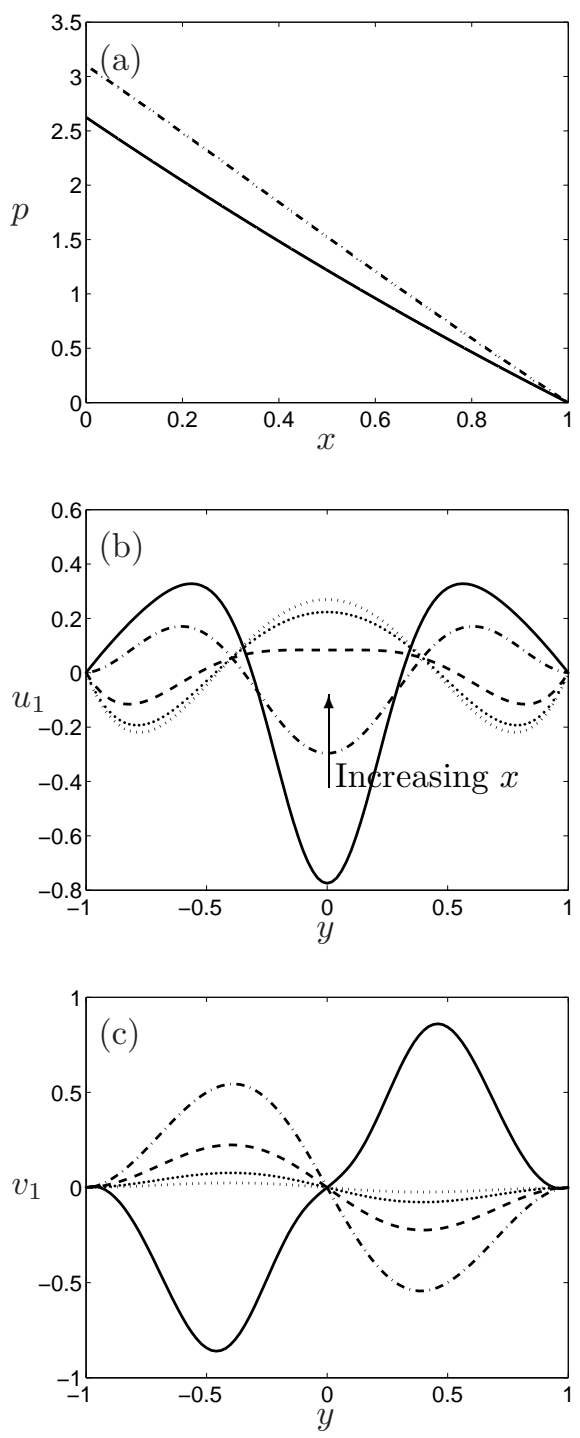

Figure 5: Pressure and order- $\epsilon$ velocity profiles given by (40) for the case of constant leakage flow through the channel walls, $\mathcal{V}^{*}=0.25$. (a) The hydrodynamic pressure, $p=p_{0}+\epsilon p_{1}$, for $\epsilon=0.05$ (dotdashed) is plotted with the leading-order term, $p_{0}$ (solid). (b) Profile of the order- $\epsilon$ axial velocity perturbation, $u_{1}$, for $x=0$ (solid), $x=0.25$ (dot-dashed), $x=0.5$ (dashed), $x=0.75$ (dotted) and $x=1$ (skinny-dotted), (c) profile of order- $\epsilon$ transverse velocity perturbation, $v_{1}$, for $x=0$ (solid), $x=0.25$ (dot-dashed), $x=0.5$ (dashed), $x=0.75$ (dotted) and $x=1$ (skinny-dotted). In the computations we have taken $P e=3, \sigma^{2}=0.05$. 


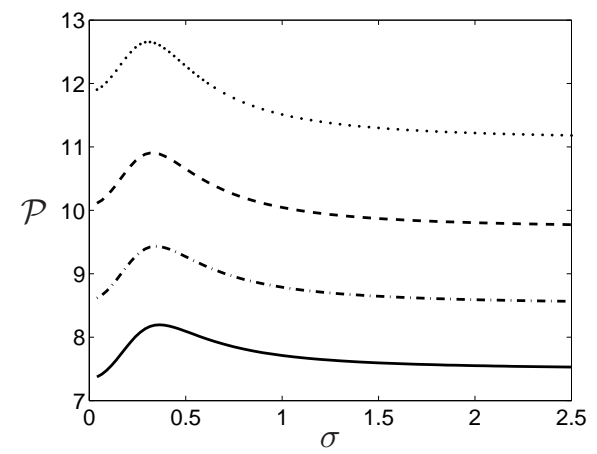

Figure 6: Variation of the pressure perturbation parameter $\mathcal{P}$ given by (46) with $\sigma$ for the case of constant leakage flow: $\mathcal{V}^{*}=0$ (solid), $\mathcal{V}^{*}=0.2$ (dot-dashed), $\mathcal{V}^{*}=0.4$ (dashed) and $\mathcal{V}^{*}=0.6$ (dotted). As $\sigma$ increases, $\mathcal{P}$ tends to a constant, with a non-trivial behaviour for $\sigma<2$ showing a critical value of $\sigma$ that maximizes $\mathcal{P}$. Note that $\mathcal{P} \rightarrow 15 / 2$ as $\sigma \rightarrow \infty$ when $\mathcal{V}^{*}=0$. In the computations, we have taken $\mathrm{Pe}=3$.

as the leakage flux increases, see Figure 7(b). The values of $\sigma$ around this pessimal pressure perturbation reflect inlet distributions that have significant volume fraction gradients across the channel. Hence the viscosity has a significant gradient in the channel, globally, and thus the particles have a greater effect on the resulting flow. It is found that both 'thinner' and 'fatter' volume fraction distributions require less additional pressure to maintain a constant fluid influx: for larger values of $\sigma$, the volume fraction distribution is more uniform and so there is less variation to the flow due to the concentration-dependent viscosity, whereas for smaller values of $\sigma$ the volume fraction is largely confined to a small region that does not significantly affect the viscosity for large regions of the channel.

In the case of no flow through the porous walls (impermeable walls, $\mathcal{V}^{*}=0$ ) there is an analytic asymptote for $\mathcal{P}$ as $\sigma \rightarrow \infty$, namely $\mathcal{P} \rightarrow 15 / 2$ (see Figure 6 , solid line). This may be calculated using the expression for $p_{0}$ from (36) and the equation for $\mathcal{P}(46)$, since in the limit $\sigma \rightarrow \infty, \phi \equiv 1$. However, in the case of porous walls, concentration polarization along the channel walls results in $\phi_{1}$ no longer being spatially independent in the channel. This precludes the analytical calculation of the asymptote that is observed numerically in the limit $\sigma \rightarrow \infty$, see Figure $7(\mathrm{~b})$. The asymptotic value of $\mathcal{P}$ increases with the magnitude of the leakage flow.

Although the leading-order hydrodynamic pressure, $p_{0}$, required to maintain a constant influx decreases with increasing leakage flow, it is observed in Figure 6 that the order- $\epsilon$ hydrodynamic pressure, $p_{1}$, increases with increasing leakage flow. This arises as a result of there being no order- $\epsilon$ leakage flow in this case. Particles aggregate at the walls because of the leading-order leakage; by increasing the leakage velocity, $\mathcal{V}^{*}$, this increases the accumulation of particles at the wall, and thus the local viscosity, so
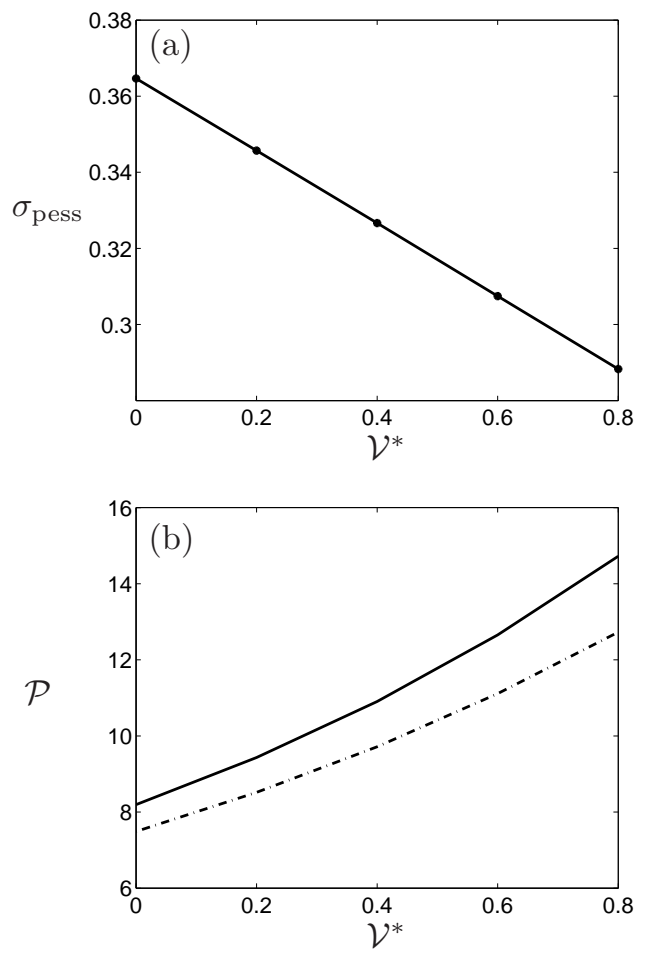

Figure 7: (a) The distribution width resulting in the pessimal pressure, $\sigma_{\text {pess }}$, and (b) the pessimal pressure perturbation, $\mathcal{P}_{\text {pess }}$ (solid), and the asymptotic pressure perturbation, $\mathcal{P}_{\text {asym }}$ (dot-dashed), as $\sigma \rightarrow \infty$, for the case of a constant leakage flow $\mathcal{V}=\mathcal{V}^{*}$. In the computations, we have taken $P e=3$.

more pressure is required to advect these particles along the channel.

\subsubsection{Case 2: Pressure-dependent leakage}

When there is a pressure-dependent flow through the porous channel walls, the order- $\epsilon$ leakage flow through the walls is given by $\mathcal{V}_{1}=\kappa\left[p_{1}-\pi_{0} \phi_{1}(x, y=1)\right]$. The ODE for the hydrodynamic pressure, (42), then becomes

$$
\frac{\mathrm{d}^{2} p_{1}}{\mathrm{~d} x^{2}}-3 \kappa p_{1}=3 g(x)
$$

with boundary conditions (43), where

$$
g(x):=\frac{\partial}{\partial x}\left\{\frac{\mathrm{d} p_{0}}{\mathrm{~d} x} \int_{0}^{1} \frac{5}{2} \tilde{y}^{2} \phi_{1}(x, \tilde{y}) \mathrm{d} \tilde{y}\right\}-\kappa \pi_{0} \phi_{1}(x, 1) .
$$

The homogeneous adjoint problem to (47) only permits the trivial zero solution; the Fredholm Alternative Theorem [16] then implies that the ODE in (47) with boundary conditions (43) has a unique solution. This solution can be 
found using the method of variation of parameters, giving

$$
\begin{aligned}
p_{1} & =\mathcal{P} \cosh (\sqrt{3 \kappa} x) \\
+ & 3 \int_{0}^{x}\left[\cosh (\sqrt{3 \kappa}[x-\tilde{x}]) \frac{\mathrm{d} p_{0}(\tilde{x})}{\mathrm{d} \tilde{x}} \int_{0}^{1} \frac{5}{2} \tilde{y}^{2} \phi_{1}(\tilde{x}, \tilde{y}) \mathrm{d} \tilde{y}\right] \mathrm{d} \tilde{x} \\
+ & \sqrt{\frac{3}{\kappa}} \frac{\mathrm{d} p_{0}(0)}{\mathrm{d} x} \sinh (\sqrt{3 \kappa} x) \times \\
& \left(\int_{0}^{1} \int_{\tilde{y}}^{1} \frac{5}{2} y^{\prime} \phi_{1}\left(0, y^{\prime}\right) \mathrm{d} y^{\prime} \mathrm{d} \tilde{y}-\int_{0}^{1} \frac{5}{2} \tilde{y}^{2} \phi_{1}(0, \tilde{y}) \mathrm{d} \tilde{y}\right) \\
-\sqrt{3 \kappa} & \pi_{0} \int_{0}^{x} \phi_{1}(\tilde{x}, 1) \sinh (\sqrt{3 \kappa}[x-\tilde{x}]) \mathrm{d} \tilde{x},
\end{aligned}
$$

where we have imposed the boundary condition (43a) and flux condition (41). Imposing the final boundary condition (43b), we determine the relationship between the additional pressure, $\mathcal{P}$, and the particle distribution $\phi_{1}$

$$
\begin{aligned}
& \mathcal{P}=\sqrt{\frac{3}{\kappa}} \tanh \sqrt{3 \kappa} \frac{\mathrm{d} p_{0}(0)}{\mathrm{d} x} \times \\
& \left(\int_{0}^{1} \frac{5}{2} \tilde{y}^{2} \phi_{1}(0, \tilde{y}) \mathrm{d} \tilde{y}-\int_{0}^{1} \int_{\tilde{y}}^{1} \frac{5}{2} y^{\prime} \phi_{1}\left(0, y^{\prime}\right) \mathrm{d} y^{\prime} \mathrm{d} \tilde{y}\right) \\
& -3 \operatorname{sech} \sqrt{3 \kappa} \times \\
& \int_{0}^{1}\left[\cosh (\sqrt{3 \kappa}[1-\tilde{x}]) \frac{\mathrm{d} p_{0}(\tilde{x})}{\mathrm{d} \tilde{x}} \int_{0}^{1} \frac{5}{2} \tilde{y}^{2} \phi_{1}(\tilde{x}, \tilde{y}) \mathrm{d} \tilde{y}\right] \mathrm{d} \tilde{x} \\
& +\sqrt{3 \kappa} \pi_{0} \operatorname{sech} \sqrt{3 \kappa} \int_{0}^{1} \phi_{1}(\tilde{x}, 1) \sinh (\sqrt{3 \kappa}[1-\tilde{x}]) \mathrm{d} \tilde{x} .
\end{aligned}
$$

Given $\phi_{1}$ we may solve (50) for $\mathcal{P}$ and then the pressure $p_{1}$ and the order- $\epsilon$ velocities $u_{1}$ and $v_{1}$ follow immediately from (49) and (40).

We illustrate the resulting behaviour by considering the injection of particles with distribution width $\sigma=\sqrt{0.05}$, so that $\phi_{1}$ is as shown in Figure 4(c). The behaviour of $p_{0}$ and $p_{1}$ and the osmotic pressure are then as shown in Figure $8(\mathrm{a})$. We observe that the hydrodynamic pressure perturbation, $p_{1}$, is positive corresponding to an increase in the total hydrodynamic pressure in the channel. However, there is an axial position beyond which the osmotic pressure, $\Delta \pi$, exceeds the hydrodynamic pressure perturbation. This is unavoidable since $p_{1}=0$ at $x=1$, and this has an impact on the leakage flow observed.

The flow perturbation $u_{1}$ is shown in Figure 8(b). Near the entrance to the channel $u_{1}$ is negative in the centre of the channel where there is a high volume fraction so the total axial flow is lower than the leading-order (particlefree) flow; in regions of low particle volume fraction $u_{1}$ is positive and so the total axial flow is greater than in the absence of particles, similar to the case of constant leakage flow. However, further down the channel $u_{1}$ is negative across the entire channel. This is due to the order- $\epsilon$ transverse leakage flow which causes fluid to be removed from the channel. In this case, the perturbation to the transverse velocity, $v_{1}$, (see Figure $8(\mathrm{c})$ ) increases the total transverse velocity towards the walls over the entire
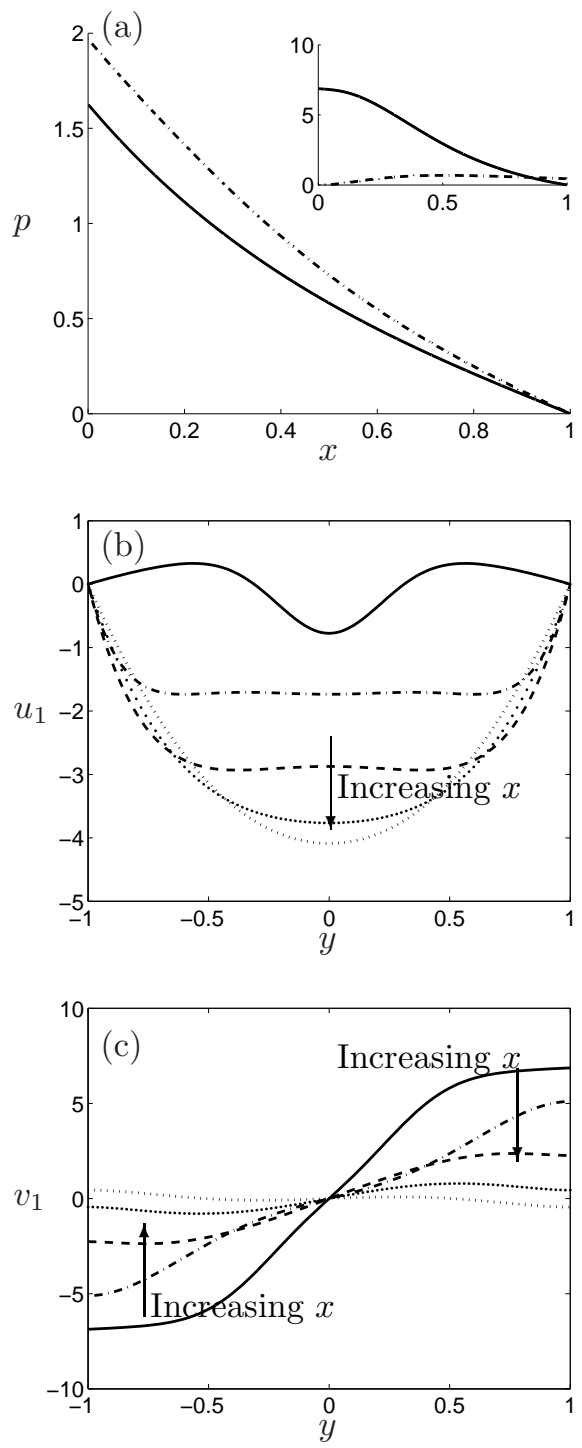

Figure 8: Pressure and order- $\epsilon$ velocity profiles given by (40) for the case of pressure-dependent leakage flow through the channel walls, $\mathcal{V}_{1}=\kappa\left[p_{1}-\pi_{0} \phi_{1}(x, 1)\right]$. (a) The hydrodynamic pressure, $p=p_{0}+\epsilon p_{1}$ for $\epsilon=0.05$ (dot-dashed) is plotted with the leadingorder term, $p_{0}$ (solid). The inset shows the pressure perturbation, $p_{1}$ (solid), and the osmotic pressure (dot-dashed) in the channel. (b) Profile of order- $\epsilon$ axial velocity perturbation, $u_{1}$, at $x=0$ (solid), $x=0.25$ (dot-dashed), $x=0.5$ (dashed), $x=0.75$ (dotted) and $x=1$ (skinny-dotted), (c) profile of order- $\epsilon$ transverse velocity perturbation, $v_{1}$, at $x=0$ (solid), $x=0.25$ (dot-dashed), $x=0.5$ (dashed), $x=0.75$ (dotted) and $x=1$ (skinny-dotted). In the computations, we have taken $P e=3, \sigma^{2}=0.05$. 


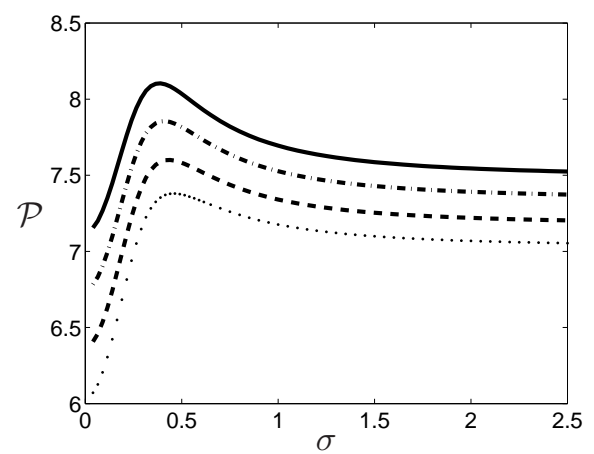

Figure 9: Variation of the pressure perturbation parameter, $\mathcal{P}$, given by (50) with $\sigma$ for the case of pressure-dependent leakage flow through the channel walls, $\mathcal{V}_{1}=\kappa\left[p_{1}-\pi_{0} \phi_{1}(x, 1)\right]$ with $\kappa=0.25$ (solid), $\kappa=0.5$ (dot-dashed), $\kappa=0.75$ (dashed) and $\kappa=1$ (dotted). As $\sigma$ increases $\mathcal{P}$ tends to a constant, again with a non-trivial behaviour for $\sigma<2$ showing a critical $\sigma$ to at which $\mathcal{P}$ is maximized. In the computations, we have taken $P e=3$.

length of the channel. This induces an additional fluid flux through the channel walls which also advects particles towards the walls, increasing the osmotic pressure due to concentration polarization up to the point where it exceeds the hydrodynamic pressure (see Figure 8(a)). This excess osmotic pressure results in a net inward flow (classic osmosis), which is undesirable in filtration because it reduces the amount of pure water that is produced by filtration. Hence, near the end of the channel this osmotic inflow is unavoidable, when $p_{\text {outer }}=0$. However, since the particles in the channel do not affect the leading-order flow, there is no leading-order osmotic inflow of fluid, and the outflow through the channel walls tends to zero at the end of the channel (since $p=0$ at $x=1$ ). Inflow of fluid by osmosis is therefore an order- $\epsilon$ effect.

The relationship between $\mathcal{P}$ and $\sigma$ is shown in Figure 9 for different constants of proportionality, $\kappa$, for the pressure-dependent leakage flow. We see a similar functional relationship to Case 1 for constant leakage, specifically the existence of a pessimal distribution of particles as shown. In this case there is no simple analytical asymptote as $\sigma \rightarrow \infty$, for the same reasons as in Case 1 with $\mathcal{V}^{*} \neq 0$. The value of the distribution width resulting in the pessimal pressure perturbation clearly increases linearly with $\kappa$, as in Figure 10(a). However, the pessimal pressure perturbation is observed to decrease with $\kappa$, in an approximately linear fashion (provided $\kappa$ exceeds a certain value, $\kappa \approx 0.3$ ) as in Figure 10(b). Similarly to Case 1, the leading-order hydrodynamic pressure, (39), in the channel is reduced by the leakage velocity. However, this case differs in that the asymptote for $\mathcal{P}$ as $\sigma \rightarrow \infty$ decreases as the leakage flow through the channel walls increases through an increase in wall permeability in $\kappa$. This decrease is approximately linear for $\kappa>0.3$, as in Figure 10(b). Hence, less pressure is required to ensure a constant influx of fluid for higher wall permeabilities when particles are present in the chan-
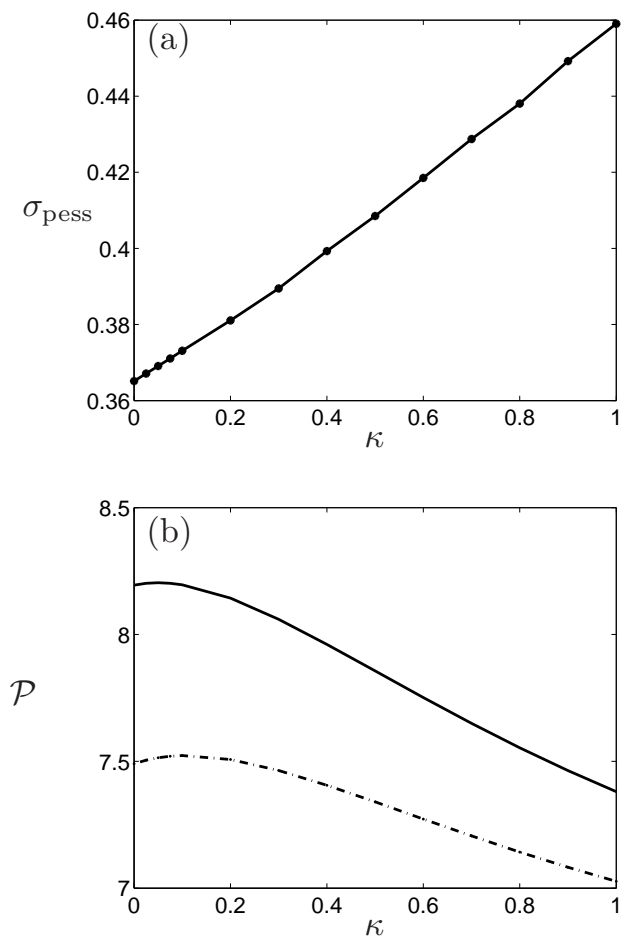

Figure 10: (a) The distribution width resulting the the pessimal pressure, $\sigma_{\text {pess }}$, and (b) the pessimal pressure perturbation, $\mathcal{P}_{\text {pess }}$ (solid), and the asymptotic pressure perturbation, $\mathcal{P}_{\text {asym }}$ (dotdashed), as $\sigma \rightarrow \infty$, for different channel wall permeabilities, $\kappa$. In the computations, we have taken $P e=3$.

nel. This result is a consequence of the osmotic component of $\mathcal{P}$, which becomes increasingly negative with $\kappa$ and so causes the reduction in the pressure perturbation. This suggests that the component of the channel for which there is a net fluid outflow and which acts as an effective filter is shorter because more fluid is lost through the walls earlier in the channel, reducing the hydrodynamic pressure, $p$, required to maintain a given fluid influx.

\subsection{Total Permeate Flux}

A quantity of particular interest is the total flux of fluid that flows out through the porous walls, $F$. This is given by the integral of the transverse velocity along the wall,

$$
\begin{aligned}
F & =2 \int_{0}^{1} v(x, 1) \mathrm{d} x, \\
& =2 \int_{0}^{1} v_{0}(x, 1)+\epsilon v_{1}(x, 1) \mathrm{d} x=F_{0}+\epsilon F_{1} .
\end{aligned}
$$

(Here the factor of two is due to the symmetry of the system about $y=0$.) This is easily calculated for the constant leakage flow case since here $\mathcal{V}=\mathcal{V}^{*}$ for all $0 \leq x \leq 1$, and so the total fluid flux passing through the porous walls is $F=2 \mathcal{V}^{*}$.

For the pressure-dependent leakage flow case, the leading-order leakage flow may also be calculated analytically. At leading order the leakage flow is given by $\kappa p_{0}$ 

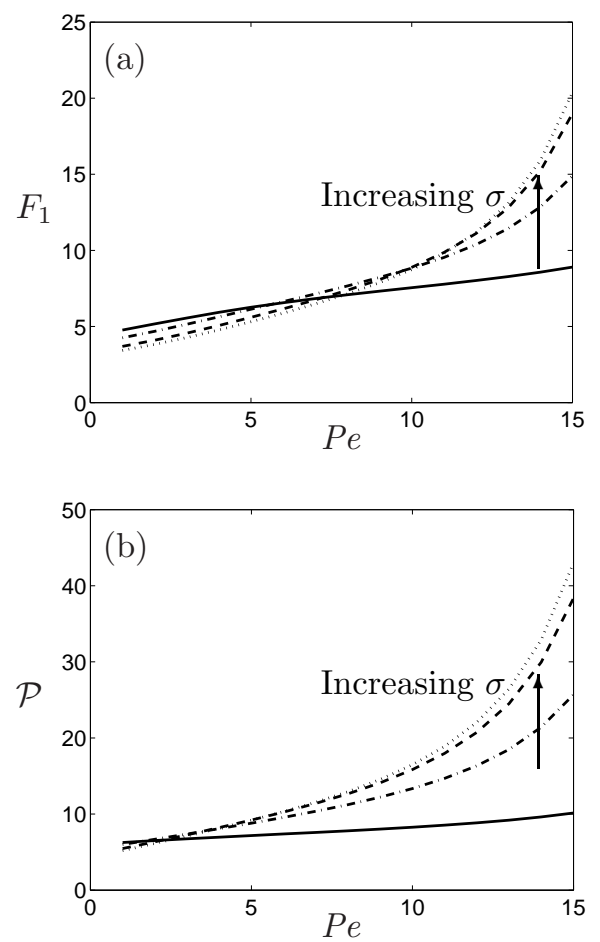

Figure 11: (a) Order- $\epsilon$ flux through the channel walls as a function of Péclet number, $P e$, for $\sigma=0.2$ (solid), $\sigma=0.4$ (dot-dashed), $\sigma=0.75$ (dashed), and $\sigma=1.5$ (skinny-dotted). (b) Pressure perturbation parameter, $\mathcal{P}$, against Péclet number for $\sigma=0.2$ (solid), $\sigma=0.4$ (dot-dashed), $\sigma=0.75$ (dashed), and $\sigma=1.5$ (skinnydotted).

where $p_{0}$ is given in equation (39). The total flux due to the leading-order term is thus $F_{0}=2(1-\operatorname{sech} \sqrt{3 \kappa})$, an increasing function of $\kappa$ as might be expected. However, the order- $\epsilon$ term must be calculated numerically. The interesting feature of the order- $\epsilon$ term, $F_{1}$, is that it is dependent on the particles in the flow. In addition, there is an osmotic inflow of fluid towards the end of the channel that reduces the net permeate flux. Consequently, both the inlet distribution width, $\sigma$, and the Péclet number, $P e$, influence the result (Figure 11(a)). For lower Péclet numbers, more localized distributions (lower values of $\sigma$ ) result in larger fluxes, but for larger Péclet numbers, more spatially uniform distributions (larger values of $\sigma$ ) produce larger fluxes. Analysing the pressure perturbation parameter, $\mathcal{P}$, with Péclet number (Figure 11(b)), we see that, as the Péclet number is increased, a greater pressure is required to maintain a constant influx. Since an increase in Péclet number also increases the leakage flux, this suggests a direct correspondence between hydrodynamic pressure and leakage flux, as one would expect.

\section{Pressure Outside the Channel}

In the previous section we concluded that it is an inevitable consequence of the osmotic pressure that an $\mathcal{O}(\epsilon)$ flux of fluid enters the channel through its walls in a pressure-dependent leakage flow. In water filtration, this effect, at any order, is undesirable; here we consider a modified set-up that eliminates this inflow.

The modification we consider is the case where the pressure outside the channel, $p_{\text {outer }}$, is a non-zero constant. The effective total transmembrane pressure may then be written as

$$
\Delta p-\Delta \pi=p_{0}(x)-p_{\text {outer }}+\epsilon\left[p_{1}(x)-\pi_{0} \phi_{1}(x, 1)\right] .
$$

We retain, without loss of generality, the condition that the fluid pressure, $p$, is zero at the end of the channel. In the previous section we found that if $p_{\text {outer }}=0$ then towards the end of the channel the fluid pressure falls below the osmotic pressure so that the effective transmembrane pressure is negative, resulting in fluid entering the channel through the membrane. However, we may choose $p_{\text {outer }}$ in such a way that the effective total transmembrane pressure remains non-negative over the entire length of the channel.

As in $\$ 4.3 .2$, osmotic pressure (order- $\epsilon$ ) is negligible in the leading-order problem (32) and the leading-order leakage flow is given here by $\mathcal{V}_{0}=\kappa\left(p_{0}-p_{\text {outer }}\right)(c f$., equation (32)). The leading-order transverse flow at $y=1(34)$ gives an ODE for the leading-order pressure

$$
\frac{1}{3} \frac{\mathrm{d}^{2} p_{0}}{\mathrm{~d} x^{2}}=\kappa\left(p_{0}-p_{\text {outer }}\right)
$$

This ODE with boundary conditions (35) has the solution

$$
\begin{aligned}
p_{0}(x) & =p_{\text {outer }}-\sqrt{\frac{3}{\kappa}} \sinh (\sqrt{3 \kappa} x) \\
& +\left(\sqrt{\frac{3}{\kappa}} \tanh \sqrt{3 \kappa}-p_{\text {outer }} \operatorname{sech} \sqrt{3 \kappa}\right) \cosh (\sqrt{3 \kappa} x) .
\end{aligned}
$$

The leading-order velocities $\left(u_{0}, v_{0}\right)$ may be calculated by substituting (54) into equation (33). These, in turn, may be used to calculate the volume fraction of particles, $\phi_{1}$, in the channel by solving the advection-diffusion equation $(12 \mathrm{~d})$.

If $p_{\text {outer }}>0$ then the pressure difference across the membrane is reduced, reducing the leakage flux which in turn increases the required hydrodynamic pressure. Since the leakage flux is reduced, concentration polarization at the channel walls is reduced. However, as $p=0$ at $x=1$, there is a point in the channel at which $p<p_{\text {outer }}$ and so there is an induced leading-order leakage influx from the outside into the channel through the channel walls. This inflow does not occur at leading order with $p_{\text {outer }}=0$. Conversely, if $p_{\text {outer }}<0$ then the pressure difference across the membrane is increased, increasing the leakage flux which in turn decreases the required hydrodynamic pressure. Here we do not have an influx of fluid into the channel at any position at leading order if $p_{\text {outer }}$ is larger in magnitude than the osmotic pressure (order- $\epsilon$ ). However, since the leakage flux is increased, there is a greater concentration-polarization effect at the channel walls. 
The leading-order total permeate flux, $F_{0}$, for a nonzero $p_{\text {outer }}$, reads

$$
\begin{aligned}
F_{0}=\frac{2}{3} \sqrt{\kappa}\left[\frac{3}{\sqrt{\kappa}}(1\right. & -\operatorname{sech} \sqrt{3 \kappa}) \\
& \left.-\sqrt{3} p_{\text {outer }} \tanh \sqrt{3 \kappa}\right]
\end{aligned}
$$

using (51). Setting $p_{\text {outer }}=0$ we retrieve the expression for $F_{0}$ from $\S 4.4$. We note that $F_{0}$ is a decreasing function of $p_{\text {outer }}$ for all $\kappa$. Furthermore, the order- $\epsilon$ total permeate flux, $F_{1}$, is an increasing function of the outside pressure: for $p_{\text {outer }}=0.25, F_{1}$ is less than that with zero outer pressure for all $P e$ and $\sigma$; conversely, for $p_{\text {outer }}=-0.25, F_{1}$ is greater than that with zero outer pressure for all $P e$ and $\sigma$.

By setting $p_{\text {outer }}<0$ we ensure a number of outcomes. Firstly, no fluid leaks into the channel from the walls. From a water-filtration perspective this means that none of the filtered water re-enters the channel. Secondly, we increase the leakage velocity at the walls resulting in more fluid being filtered. The penalty we pay in doing so is the extra energy required to generate the negative external pressure.

\subsection{Order- $\epsilon$ outer pressure}

We wish to choose the outer pressure to avoid any reentry of fluid into the channel through the walls. In the previous section we saw that this might be achieved by using a sufficiently large negative outer pressure. However, we also want to minimize the energy required to generate this additional pressure as so it is the 'optimum' outer pressure that is of interest. Since re-entry is an order- $\epsilon$ effect due to the osmotic pressure, we assume that $p_{\text {outer }}$ is $\mathcal{O}(\epsilon)$. Also, since $F_{0}$ differs between the cases of an outer pressure and no outer pressure, a better comparison of how the particles affect the flow is to consider an outer pressure that is of order $\epsilon$. Now the leading-order pressure is given by equation (39) and $F_{0}$ does not depend on $p_{\text {outer }}$ and remains as in the case of $p_{\text {outer }}=0$, that is, $F_{0}=2(1-\operatorname{sech} \sqrt{3 \kappa})$.

As the leading-order problem remains unchanged, and the re-entry is a result of the osmotic pressure exceeding the hydrodynamic pressure near the exit of the channel, we take $p_{\text {outer }}$ to be equal and opposite to the osmotic pressure at the exit of the channel, that is

$$
p_{\text {outer }}=-\epsilon \pi_{0} \phi_{1}(1,1) \text {. }
$$

The transmembrane pressure difference now reads

$$
\Delta p-\Delta \pi=p_{0}(x)+\epsilon\left\{p_{1}(x)-\pi_{0}\left[\phi_{1}(x, 1)+\phi_{1}(1,1)\right]\right\} .
$$

This leads to a modification to the order- $\epsilon$ pressure given by equation (49) and the pressure perturbation given by equation (50). In this case the terms $\phi_{1}(\tilde{x}, 1)$ in the final term of each equation are replaced with $\phi_{1}(\tilde{x}, 1)-\phi_{1}(1,1)$.

The choice of outer pressure that negates the osmotic effect does indeed prevent any influx of fluid from outside
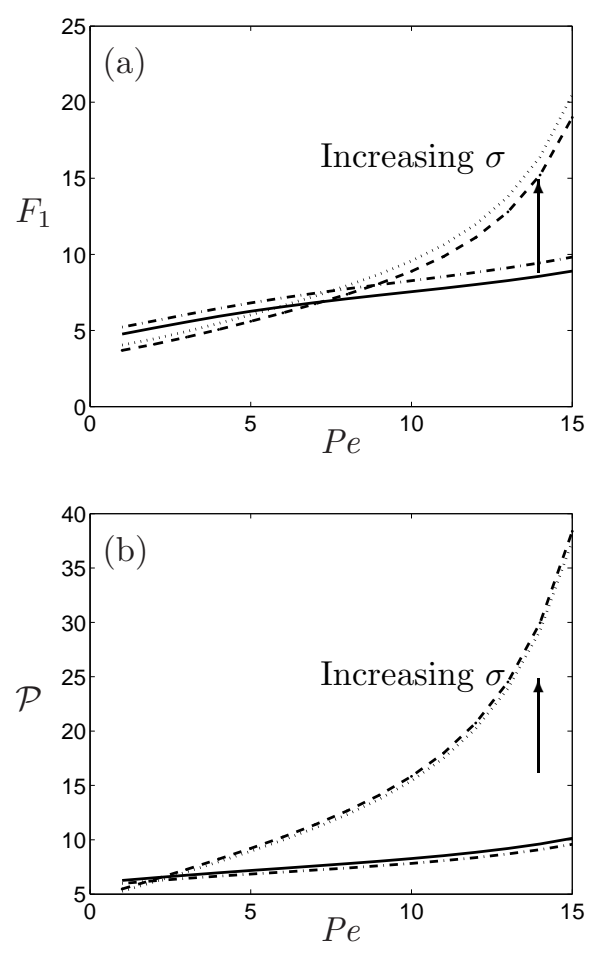

Figure 12: (a) Order- $\epsilon$ flux through the channel walls as a function of Péclet number, $P e$, for $\sigma=0.2$ with zero outer pressure (solid) and an order- $\epsilon$ outer pressure $p_{\text {outer }}=-\pi_{0} \phi_{1}(1,1)$ (dotdashed), and $\sigma=0.75$ (dashed, skinny-dotted respectively). We see that the order- $\epsilon$ outer pressure increases the fluid flux. (b) Pressure perturbation parameter, $\mathcal{P}$, as a function of Péclet number for $\sigma=0.2$ with zero outer pressure (solid) and an order- $\epsilon$ outer pressure $p_{\text {outer }}=-\pi_{0} \phi_{1}(1,1)$ (dot-dashed), and $\sigma=0.75$ (dashed, skinny-dotted respectively). We see that the order- $\epsilon$ outer pressure decreases the pressure perturbation parameter.

the channel through the walls, with transverse velocity giving a leakage flux out of the channel at each point along the wall. This results in a greater order- $\epsilon$ permeate flux $F_{1}$ as seen in Figure 12(a) as well as a reduction in the pressure perturbation $\mathcal{P}$ as in Figure 12(b).

By choosing $p_{\text {outer }},(56)$, in this way we ensure that no fluid leaks into the channel from the walls. However, in terms of the energy penalty we pay in doing so, the analysis here provides a mechanism for determining the minimum suction pressure required to ensure that no filtered fluid re-enters the channel, thus optimizing the filtration operation if the actual outlet gauge is zero $(p=0$ at $x=0)$. For a positive outlet pressure, $p_{\text {outer }}$ could be zero or even positive.

\section{Conclusion}

The flow and particle distribution for a dilute suspension in a channel flow with porous walls has been described. The presence of particles reduces the flow velocity by increasing the viscosity of the fluid. Allowing leakage (either constant or pressure dependent) through the porous walls reduces the pressure required for the fluid 
to flow at a constant influx. This leakage flow also contributes to the transport of particles from the centre of the channel to the channel walls, leading to concentration polarization when the walls are impermeable to particles.

We considered the effect of varying the width of an inlet pulse of particles in the cross-stream channel direction. Beginning with a very narrow pulse (relatively highly concentrated in a small region around the centre of the channel), we observe that it initially becomes harder to push the particles as this pulse width increases, that is, a higher inlet hydrodynamic pressure is required. We attribute this to the size of the region of the channel occupied by the particles: for small widths the particles do not affect a significant region of the channel to alter the fluid flow; however, as this pulse width increases, the particles affect a greater proportion of the fluid and so a greater pressure is required to transport the particles with a constant fluid influx. Interestingly, this increase in pressure reverses as the width of the inlet distribution of particles surpasses a critical value and it becomes easier (requires less pressure) to transport the particles. We attribute this observation to the lower particle volume fraction gradients in the channel, since the particles are now more uniformly distributed. Generally, we observe a critical, pessimal value of the inlet distribution width that maximizes the additional pressure required. Since this inlet distribution requires the greatest pressure to maintain a constant fluid influx, the energy required and total cost of pumping the fluid-particle mixture into the channel is maximized - this suggests that filtration systems should avoid the intermediate distribution widths. As we increase the width of the distribution, approaching the case of a uniform distribution at the inlet, the additional pressure required tends to a constant asymptote in all cases.

In the case of pressure-dependent leakage flow, concentration polarization results in the osmotic pressure exceeding the hydrodynamic pressure at the end of the channel and an inward flow from the porous channel walls. In a filtration system, this situation of pure fluid entering the channel contaminated by particles is undesirable yet unavoidable due to the condition of equal outlet pressure and pressure outside the channel.

Finally, we analysed the effect of a constant outer pressure to the channel walls. This appears through the transmembrane pressure difference. A positive outer pressure decreases concentration polarization at the channel walls but again there is an unavoidable influx of fluid through the channel walls. A negative outside pressure increases the leakage flux, with no influx, but at the cost of increased concentration polarization, and energy expenditure. Taking an outer pressure that is equal and opposite to the osmotic pressure at the end of the channel negates the influx caused by osmosis. Since the magnitude of the outer pressure is small, it is a reasonable compromise in terms of additional energy required to prevent the re-entry of fluid into the channel.

Our results here thus offer two routes to design opti- mization via the minimization of energy required: first, insight into particle distributions that should be avoided, and second, guidance on the appropriate external pressure to avoid the re-entry of filtered water. Both features give new insight into strategies employed in cross-flow filtration devices.

\section{Appendix A. Numerical scheme}

We exploit symmetry about the $y$-axis to consider the domain $(x, y) \in[0,1] \times[0,1]$, and discretize this domain by introducing grid points

$$
\begin{array}{ll}
x_{i}=h i, & i=0,1 \ldots N, \\
y_{j}=k j, & j=0,1 \ldots M,
\end{array}
$$

for grid spacings $h$ and $k$ where $h=\Delta x=1 / N$ and $k=\Delta y=1 /(M-1)$. We index the variable $\phi$ as follows

$$
\phi_{i, j}=\phi_{1}\left(x_{i}, y_{j}\right) \text {. }
$$

The resulting solutions are then mapped to the other half of the domain, $-1 \leq y<0$. A forward-centre finite difference scheme for advection-diffusion equation (27) is

$$
\begin{gathered}
\phi_{i+1, j}=\phi_{i, j} \\
+\frac{2 h}{p^{\prime}\left(x_{i}\right)\left(y_{j}^{2}-1\right)}\left(-p^{\prime \prime}\left(x_{i}\right) \frac{3 y_{j}-y_{j}^{3}}{6} \frac{\phi_{i, j+1}-\phi_{i, j-1}}{2 k}\right. \\
\left.\quad+\frac{1}{P e} \frac{\phi_{i, j+1}-2 \phi_{i, j}+\phi_{i, j-1}}{k^{2}}\right) \\
\phi_{i+1,1}=\phi_{i, 1}-\frac{2 h}{p^{\prime}\left(x_{i}\right)}\left(\frac{2}{P e} \frac{\phi_{i, 2}-\phi_{i, 1}}{k^{2}}\right) \\
\phi_{i+1, M}=\frac{2 \phi_{i+1, M-1}-\frac{1}{2} \phi_{i+1, M-2}}{\frac{3}{2}-k P e v_{i+1, M}} \\
\phi_{1, j}=\Phi\left(y_{j}\right)
\end{gathered}
$$

for $i=1,2, \ldots N-1$ and $j=2,3, \ldots M-1$. The standard forward-centre finite difference discretization for nonboundary points of the advection-diffusion equation (27) is given in (A.3a), with the symmetry condition $\partial \phi_{1} / \partial y=0$ at $y=0$ given by (A.3b) and the no-flux boundary condition given by (A.3c). There is an initial condition of some $\phi(0, x)=\Phi(\mathrm{A} .3 \mathrm{~d})$. The scheme (A.3) is second-order accurate.

For a constant-coefficient convection-diffusion equation

$$
f_{x}+a f_{y}=b f_{y y}
$$

with $b>0$, there are two mesh size parameters to consider

$$
\nu=\frac{a \Delta x}{\Delta y}, \quad \eta=b \frac{\Delta x}{\Delta y^{2}} .
$$

Implementing a stable forward in $x$, central-differences in $y$ finite-differences scheme, it is required that [17]

$$
0<\nu \leq 1, \quad 0<\eta \leq \frac{1}{2}
$$


In our governing equations, the advection-diffusion equation $(12 \mathrm{~d})$ for the volume fraction $\phi_{1}$ does not have constant coefficients, but the coefficients are well behaved, with no singularities. We ensure that the scheme is stable by requiring $\Delta x / \Delta y^{2}=1 / 50 \ll 1 / 2$ for stability: we use $\Delta y=2 \times 10^{-2}$ and $\Delta x=8 \times 10^{-6}$.

\section{Acknowledgements}

This publication is based on work supported by Award No. KUK-C1-013-04, made by King Abdullah University of Science and Technology (KAUST).

\section{References}

\section{References}

[1] L. Song, M. Elimelech, Theory of concentration polarization in crossflow filtration, J. Chem. Soc. Faraday. Trans. 91 (1995) 3389-3398.

[2] R. F. Probstein, Physicochemical Hydrodynamics. An Introduction, Butterworths, 1989.

[3] M. Kostoglou, A. J. Karabelas, Mathematical analysis of fluid flow and mass transfer in a cross flow tubular membrane, Ind. Eng. Chem. Res. 48 (2009) 5885-5893.

[4] R. H. Davis, J. D. Sherwood, A similarity solution for steadystate crossflow microfiltration, Chem. Eng. Sci. 45 (1990) 32033209.

[5] R. H. Davis, D. T. Leighton, Shear-induced transport of a particle layer along a porous wall, Chem. Eng. Sci. 42 (1987) 275-281.

[6] W. R. Bowen, P. M. Williams, Prediction of the rate of crossflow ultrafiltration of colloids with concentration-dependent diffusion coefficient and viscosity - theory and experiment, Chem. Eng. Sci. 56 (2001) 3083-3099.

[7] P. Bacchin, D. Si-Hassen, V. Starov, M. J. Clifton, P. Aimar, A unifying model for concentration polarization, gel-layer formation and particle deposition in cross-flow membrane filtration of colloidal suspensions, Chem. Eng. Sci. 57 (2002) 77-91.

[8] R. M. Sandblom (Alfa-Laval), Filtering Process, Patent (August 1978).

[9] G. S. Beavers, D. D. Joseph, Boundary conditions at a naturally permeable wall, J. Fluid Mech. 30 (1967) 197-207.

[10] R. J. Shipley, S. L. Waters, M. J. Ellis, Definition and Validation of Operating Equations for Poly(Vinyl Alcohol)-Poly(LactideCo-Glycolide) Microfiltration Membrane-Scaffold Bioreactors, Biotech. Bioeng. 107 (2010) 382-392.

[11] F. A. Williams, A nonlinear diffusion problem relevant to desalination by reverse osmosis, SIAM J. Appl. Math. 17 (1969) 59-73.

[12] E. Guazzelli, J. F. Morris, A Physical Introduction to Suspension Dynamics, Cambridge University Press, 2012.

[13] M. Elimelech, J. Gregory, X. Jia, R. A. Williams, Particle Deposition and Aggregation. Measurement, Modelling and Simulation, Butterworth-Heinemann, 1995.

[14] G. K. Batchelor, Brownian diffusion of particles with hydrodynamic interaction, J. Fluid Mech. 74 (1976) 1-29.

[15] R. W. Field, G. K. Pearce, Critical, sustainable and threshold fluxes for membrane filtration with water industry applications, Adv. Colloid Interface Sci. 164 (2011) 38-44.

[16] J. P. Keener, Principles of Applied Mathematics, Westview, 2000.

[17] K. W. Morton, D. F. Mayers, Numerical Solution of Partial Differential Equations, Cambridge University Press, 2005. 



\section{RECENT REPORTS}

12/110 Multiscale stochastic reaction-diffusion modelling: application to actin dynamics in filopodia

Erban

Flegg

Papoian

12/111 Exploiting the Synergy Between Carboplatin and ABT-737 in the Treatment of Ovarian Carcinomas

Jain

Richardson

Meyer-Hermann

Byrne

12/112 The integration of hormonal signaling networks and mobile microRNAs is required for vascular patterning in Arabidopsis roots

Muraro

Pound

Help

Lucas

Chopard

Byrne

Godin

Hodgman

King

Pridmore

Helariutta

Bennett

Bishopp

12/113 Fast solution of Cahn-Hilliard Variational Inequalities using Implicit Time Discretization and Finite Elements

Bosch

Stoll

Benner

12/114 An Embedding Technique for the Solution of Reaction-Diffusion Equations on Algebraic Surfaces with Isolated Singularities

Rockstroh

März

Ruuth

12/115 Mathematicians at the Movies: Sherlock Holmes vs. Professor

Moulton Moriarty

Goriely

13/01 Rotation, inversion, and perversion in anisotropic elastic cylindrical tubes and membranes

Goriely

Tabor

13/02 Drop spreading and penetration into pre-wetted powders

Marston

Sprittles

Zhu

Li

Vakarelski

Thoroddsen

$13 / 03$ On the mechanics of thin films and growing surfaces

Holland

Kosmata

Goriely

Kuhl

Ketcheson

Macdonald

Ruuth

13/05 Simple computation of reaction-diffusion processes on point

Macdonald clouds 
13/10 Capillary deformations of bendable films

Schroll

Adda-Bedia

Cerda

Huang

Menon

Russell

Toga

Vella

Davidovitch

13/11 Twist and stretch of helices: All you need is Love

Đuričković

Goriely

Maddocks

13/12 Switch on, switch off: stiction in nanoelectromechanical switches

Wagner

Vella

13/13 Pinning, de-pinning and re-pinning of a slowly varying rivulet

Paterson

Wilson

Duffy

13/14 Travelling-wave similarity solutions for a steadily translating slender dry patch in a thin fluid film

Yatim

Duffy

Wilson

13/15 A stochastic model for early placental development

Cotter

Klika

Kimpton

Collins

Heazell

13/16 Experimentally-calibrated population of models predicts and ex-

Britton plains inter-subject variability in cardiac cellular electrophysiology

Bueno-Orovio

Van Ammel

Lu

Towart

Gallacher

Rodriguez

13/17 Elastometry of deflated capsules elastic moduli from shape and wrinkle analysis

Knoche

Vella

Aumaitre

Degen

Rehage

Cicuta

Kierfeld

\section{Copies of these, and any other OCCAM reports can be obtained from:}


Oxford Centre for Collaborative Applied Mathematics Mathematical Institute

24 - 29 St Giles'

Oxford

OX1 3LB

England

www.maths.ox.ac.uk/occam 